\title{
First results on spectrally bounded operators
}

\author{
by \\ Martin Mathieu and Gerhard J. Schick (Belfast)
}

\begin{abstract}
A linear mapping $T$ from a subspace $E$ of a Banach algebra into another Banach algebra is defined to be spectrally bounded if there is a constant $M \geq 0$ such that $r(T x) \leq M r(x)$ for all $x \in E$, where $r(\cdot)$ denotes the spectral radius. We study some basic properties of this class of operators, which are sometimes analogous to, sometimes very different from, those of bounded operators between Banach spaces.
\end{abstract}

1. Introduction. A linear operator between Banach algebras is said to be spectrally bounded if it increases the spectral radius of each element but by a universal multiplicative constant. (For the precise definition, see Section 2 below.) Spectrally bounded operators made their appearance, in various guises, in a number of papers since the mid 1970's; see [1, 8, 15, 16, 22], to cite but a few. The concept became more prominent when it was discovered that a derivation $d$ on a Banach algebra $A$ is spectrally bounded if and only if $d A$ is contained in the Jacobson $\operatorname{radical} \operatorname{rad}(A)$ of $A$ [9, Theorem 2.5]. This revealed an intimate interrelation with the non-commutative Singer-Wermer conjecture and, hence, with Automatic Continuity Theory [12]. Another instance of such an interplay had arisen earlier in Aupetit's approach to Johnson's uniqueness-of-the-complete-norm-topology theorem [1]. Aupetit proved that every surjective spectrally bounded operator onto a semisimple Banach algebra must be bounded, from which Johnson's result follows easily.

Recently, spectrally bounded operators and their properties were studied in greater detail in $[7,10,11,18]$. For instance, Šmrl proved that every bijective spectrally bounded operator on the algebra $B(H)$ of all bounded linear operators on an infinite dimensional Hilbert space $H$ is a Jordan auto-

2000 Mathematics Subject Classification: Primary 47B48; Secondary 46B99, 46H40, 47A10, 47L10.

Key words and phrases: spectral structure on Banach spaces, spectrally bounded operators, Banach algebras, automatic continuity.

This paper is part of the research in the EU network Analysis and Operators (HPRNCT-2000-00116). It was presented at the Banach Algebras in Context conference held in Odense in August 2001. 
morphism [19], and his result was extended to arbitrary properly infinite von Neumann algebras in [13]. In the formulation presented below, the concept of a spectrally bounded operator turned out to be crucial in order to extend Johnson's automatic continuity theorem for homomorphisms to Lie homomorphisms in [5]. The continuity of Lie homomorphisms had been studied before in [17] and [6] under rather restrictive assumptions.

Having thus proved its usefulness, the concept of a spectrally bounded operator deserves a thorough study from scratch, we believe. It is the purpose of the present article to establish some first results in this direction. Of course, not in the sense of being the first results proven, but rather some basic observations on which a fuller theory eventually should build. Section 2 is devoted to an introduction of the appropriate framework and a compilation of some of the existing material in a systematic way. In analogy to the theory of bounded linear operators, we then start a discussion of a duality theory with a closer look at spectrally bounded linear functionals in Section 3 below. It will emerge that, due to the failure of a general Hahn-Banach theorem, the notion of a spectral dual (see Definition 3.1) is far less powerful than the usual concept of the topological dual of a Banach space.

The fourth section is concerned with the investigation of spectral isometries (i.e., spectral radius-preserving operators) with an outlook on a possible structure theorem for them analogous to the one recently obtained for spectrally bounded operators in [13].

2. The framework for spectrally bounded operators. In this paper, we study operators defined on normed spaces that are endowed with a "spectral structure". To this end, we consider a normed space $E$, a unital Banach algebra $A$, and a linear topological isomorphism $\iota$ from $E$ into $A$. Strictly speaking, our objects are therefore triples $(E, A, \iota)$; however, in order to simplify the notation, we shall always suppress $\iota$ and consider $E$ already as a normed subspace of $A$. We denote this by writing " $E \subseteq A$ ", and we say that $E$ is a spectrally normed space; but we emphasize that the "spectral structure" on $E$ depends on the embedding, up to topological isomorphism.

In this setting, $E \subseteq A$, we can define the spectral radius $r(x)$ of $x \in E$ by the usual formula $r(x)=\lim _{n \rightarrow \infty}\left\|x^{n}\right\|^{1 / n}$, computed in $A$. Note that, once the embedding is fixed, this does not depend on whether we consider the entire algebra $A$ or any unital closed subalgebra of $A$ containing $E$. Hence, if we assume that $E \subseteq A$ is commutative, we may without loss of generality suppose that $A$ itself is commutative, upon replacing $A$ by a maximal commutative subalgebra of $A$ containing the commutative subset $E$, if necessary.

EXAMPLES 2.1. 1. Every normed space $E$ carries at least one spectral structure via the isometric embedding $j_{E}$ of $E$ into $C\left(E_{1}^{\prime}\right)$, the complex- 
valued continuous functions on the dual unit ball $E_{1}^{\prime}$ endowed with the weak*-topology. This is a commutative, semisimple structure on $E$; in fact, $\|x\|=r(x)$ for all $x \in E$ in this case.

2. Suppose that $E$ is a unital Banach algebra itself. Then there is the canonical spectral structure on $E$ given by the identical mapping. This structure coincides with the one induced by considering $E$ as a closed subalgebra of the algebra $B(E)$ of all bounded linear operators on $E$ via the left regular representation.

Definition 2.2. Let $E \subseteq A, F \subseteq B$ be spectrally normed spaces. A linear mapping $T: E \rightarrow F$ is called spectrally bounded if there is a constant $M \geq 0$ such that

$$
r(T x) \leq M r(x) \quad(x \in E) .
$$

By $\operatorname{SB}(E, F)$ we denote the set of all spectrally bounded operators from $E$ into $F$.

Note that, if $F$ is not commutative, $\operatorname{SB}(E, F)$ will in general not be a vector space. Nevertheless we introduce an analogue of the operator norm for spectrally bounded operators.

Definition 2.3. Let $T \in \mathrm{SB}(E, F)$ for some spectrally normed spaces $E$ and $F$. We put

$$
\|T\|_{\sigma}=\inf \{M \geq 0 \mid r(T x) \leq M r(x) \text { for all } x \in E\}
$$

and call $\|T\|_{\sigma}$ the spectral operator norm of $T$.

Here are some immediate properties of the spectral operator norm.

Proposition 2.4. Suppose that $E, F, G$ are spectrally normed spaces, and let $S, T \in \mathrm{SB}(E, F)$ and $R \in \mathrm{SB}(F, G)$. Then

(i) $\|T\|_{\sigma}=\sup \{r(T x) \mid x \in E, r(x) \leq 1\}$

$$
=\sup \{r(T x) \mid x \in E, r(x)=1\}
$$

(ii) $\|\lambda T\|_{\sigma}=|\lambda| \cdot\|T\|_{\sigma}$ for all $\lambda \in \mathbb{C}$;

(iii) $\|R T\|_{\sigma} \leq\|R\|_{\sigma}\|T\|_{\sigma}$;

(iv) $\|S+T\|_{\sigma} \leq\|S\|_{\sigma}+\|T\|_{\sigma}$ if $F$ is commutative.

Proof. Let $M \geq 0$ be such that, for all $x \in E, r(T x) \leq M r(x)$. Then $\sup \{r(T x) \mid x \in E, r(x) \leq 1\} \leq M$, so the supremum is also dominated by $\|T\|_{\sigma}$. Let $\alpha=\sup \{r(T x) \mid x \in E, r(x)=1\}$ (with the understanding that $\alpha=0$ if the set on the right hand side is empty). For all quasi-nilpotent $x \in E$ certainly $r(T x) \leq \alpha r(x)$ holds since $T$ is spectrally bounded. Suppose $x \in E$ is not quasi-nilpotent. Then $r(T(x / r(x))) \leq \alpha$. Hence, $r(T x) \leq \alpha r(x)$ holds for all $x \in E$, which shows that $\alpha \geq\|T\|_{\sigma}$. Combining these facts yields assertion (i). 
Assertion (ii) follows directly from (i). Note that (i) in particular implies that $r(T x) \leq\|T\|_{\sigma} r(x)$ for all $x \in E$. Therefore,

$$
r(R T x) \leq\|R\|_{\sigma} r(T x) \leq\|R\|_{\sigma}\|T\|_{\sigma} r(x) \quad(x \in E),
$$

which entails (iii). Suppose that $F$ is commutative. Then the spectral radius is subadditive and therefore the sum of two spectrally bounded operators is spectrally bounded. Assertion (iv) is thus evident.

Notation. For a spectrally normed space $E \subseteq A$, we put $\operatorname{rad}(E)=$ $E \cap \operatorname{rad}(A)$ and we call $E$ semisimple if $\operatorname{rad}(E)=0$. This terminology is justified as we can embed $E / \operatorname{rad}(E)$ into $A / \operatorname{rad}(A)$ under preservation of the spectral structure.

COROLlary 2.5. Suppose that $F$ is a semisimple commutative spectrally normed space. For every spectrally normed space $E,\left(\mathrm{SB}(E, F),\|\cdot\|_{\sigma}\right)$ is a normed space. If $E=F$ then $\mathrm{SB}(E)=\mathrm{SB}(E, F)$ is a unital normed algebra.

Proof. This is immediate from Proposition 2.4 and the well known fact that the spectral radius is a norm on a semisimple commutative Banach algebra.

Before going any further let us consider a few examples.

Example 2.6. Let $A$ be a Banach algebra and $a, b \in A$. Denote by $d_{a, b}: x \mapsto x a-b x, x \in A$, the generalised inner derivation determined by $a$ and $b$. Then $d_{a, b}$ is bounded with $\left\|d_{a, b}\right\| \leq\|a\|+\|b\|$. By [11, Theorem B], $d_{a, b}$ is spectrally bounded if and only if both $a$ and $b$ belong to the centre modulo the radical, $\mathcal{Z}(A)$, of $A$. In this case, $\left\|d_{a, b}\right\|_{\sigma}=r(a-b) \leq\left\|d_{a, b}\right\|$.

ExAmple 2.7. Let $A=M_{2}(\mathbb{C})$ be the $C^{*}$-algebra of all complex $2 \times 2$ matrices. Let $f$ be the bounded linear functional on $A$ given by $f\left(\left(a_{i j}\right)\right)=$ $a_{11},\left(a_{i j}\right) \in A$. Then $f$ is not spectrally bounded since

$$
x_{n}=\left(\begin{array}{ll}
n & 1-n \\
n & 1-n
\end{array}\right)
$$

has spectral radius 1 for each $n \in \mathbb{N}$, but $f\left(x_{n}\right)=n$.

ExAmple 2.8. Let $A=C(X)$ be the $C^{*}$-algebra of all continuous complex-valued functions on a compact Hausdorff space $X$. Let $B=M_{2}(A)$ denote the $C^{*}$-algebra of all $2 \times 2$ matrices with entries in $A$. Suppose there is an unbounded linear functional $\varphi$ on $A$. Then the mapping

$$
T: A \rightarrow B, \quad a \mapsto\left(\begin{array}{cc}
a & \varphi(a) \\
0 & a
\end{array}\right),
$$

is an unbounded operator on $A$ which is spectrally bounded since $T a$ is invertible whenever $a \in A$ is invertible. 
These examples illustrate that, in general, there is no interrelation between boundedness and spectral boundedness for linear operators. The following observation is an exception.

Proposition 2.9. Let $E, F$ be spectrally normed spaces.

(i) Suppose that $E \subseteq C(X)$. Then $B(E, F) \subseteq \mathrm{SB}(E, F)$ and $\|\cdot\|_{\sigma} \leq\|\cdot\|$.

(ii) Suppose that $F \subseteq C(Y)$. Then $\mathrm{SB}(E, F) \subseteq B(E, F)$ and $\|\cdot\| \leq\|\cdot\|_{\sigma}$.

Proof. Both assertions are immediate from the fact that $\|x\|=r(x)$ for every bounded continuous function $x$.

Examples 2.7 and 2.8 show that the commutative $C^{*}$-algebras appearing in the assumptions of Proposition 2.9 cannot be replaced by arbitrary ones.

The following automatic continuity result was first proved for the case $E=A$, a Banach algebra, in [1, Theorem 1] (see also [2, Theorem 5.5.1]), and its extension to general $E$ is noted in [5, Lemma A]. We use the standard notation

$$
\mathcal{S}(T)=\left\{y \in F \mid \exists\left(x_{n}\right)_{n \in \mathbb{N}} \text { in } E, x_{n} \rightarrow 0, T x_{n} \rightarrow y\right\}
$$

for the separating space of the operator $T: E \rightarrow F$.

2.10. Aupetit's Lemma. Let $T$ be a spectrally bounded operator on a spectrally normed space $E$. Then $r(T x) \leq r(T x+y)$ for all $x \in E$ and all $y \in \mathcal{S}(T)$. Consequently, if $E$ is complete and $T$ maps onto a semisimple Banach algebra, then $T$ is bounded.

This result shows that, modulo the radical, surjectivity of a spectrally bounded operator entails boundedness. The behaviour with respect to the radical is easily controlled via the following observation.

Proposition 2.11. Let $T: E \rightarrow B$ be a spectrally bounded operator from a spectrally normed space $E$ onto a Banach algebra $B$. Then $T \operatorname{rad}(E) \subseteq$ $\operatorname{rad}(B)$.

Proof. By [3, Theorem 1], an element $a$ belongs to the radical if and only if $\sup \{r(x+\lambda a) \mid \lambda \in \mathbb{C}\}<\infty$ for all elements $x$. Let $a \in \operatorname{rad}(E)$. Then for each $y \in B$ there is $x \in E$ such that $T x=y$ and thus

$$
r(y+\lambda T a)=r(T(x+\lambda a)) \leq\|T\|_{\sigma} r(x+\lambda a)=\|T\|_{\sigma} r(x) .
$$

Therefore, the above characterisation of the radical completes the argument.

Corollary 2.12. Let $T: E \rightarrow B$ be a spectrally bounded operator from a complete spectrally normed space $E$ onto a Banach algebra $B$. Then the induced operator $\widehat{T}: E / \operatorname{rad}(E) \rightarrow B / \operatorname{rad}(B)$ is bounded and spectrally bounded with $\|\widehat{T}\|_{\sigma}=\|T\|_{\sigma}$. If $B$ is semisimple then $T$ itself is bounded, and $\|\widehat{T}\|=\|T\|$. 
It was shown in [9, Lemma 2.1] that every spectrally bounded derivation on a Banach algebra fixes the radical. Indeed, on a commutative Banach algebra, every derivation $d$ is spectrally infinitesimal, i.e., $\|d\|_{\sigma}=0$, by Thomas' theorem [21]. On the other hand, without additional assumptions spectral boundedness does not imply compatibility with the radical, as the following example shows.

EXAMPLE 2.13. Let $A$ be the unitisation of a radical Banach algebra. Take $x \in \operatorname{rad}(A)$ and $\varphi \in A^{\prime}$ such that $\varphi(x)=1$. Let $B=M_{2}(A)$. We define $T: A \rightarrow B$ by

$$
T a=\left(\begin{array}{cc}
a & \varphi(a) \\
0 & a
\end{array}\right) .
$$

Then $T$ preserves invertible elements and hence is spectrally bounded. However, since $\operatorname{rad}(B)=M_{2}(\operatorname{rad}(A)), T x$ is not contained in $\operatorname{rad}(B)$.

REMARK 2.14. In [13] it is shown that every spectrally bounded operator onto a semisimple Banach algebra preserves nilpotent elements, which however requires more sophisticated tools.

REMARK 2.15. The above discussion suggests the question whether an analogue of the open mapping theorem is valid for spectrally bounded operators. That is, is the inverse of a bijective spectrally bounded operator onto a (semisimple) Banach algebra spectrally bounded? This holds, e.g., if the range is of the form $C(X)$, by Proposition 2.9(i), but fails in general. We can simply take the canonical linear isomorphism between $\mathbb{C}^{4}$ and $M_{2}(\mathbb{C})$ as an example.

3. The spectral dual. In this section we shall introduce the spectral analogue of the dual space of a normed space and discuss a few of its properties. Naturally, the question of extensions of spectrally bounded functionals and the validity of a Hahn-Banach theorem play a central role.

By Corollary 2.5, the spectrally bounded linear functionals on a spectrally normed space $E$ form a normed space $\left(\operatorname{SB}(E, \mathbb{C}),\|\cdot\|_{\sigma}\right)$. It is therefore close at hand to introduce the following terminology.

Definition 3.1. For a spectrally normed space $E$ we call the normed space $\left(\operatorname{SB}(E, \mathbb{C}),\|\cdot\|_{\sigma}\right)$ the spectral dual of $E$ and denote it by $E^{\sigma}$.

Proposition 3.2. For every spectrally normed space E, the spectral dual $E^{\sigma}$ is a Banach space.

Proof. Let $\left(f_{n}\right)_{n \in \mathbb{N}}$ be a Cauchy sequence in $E^{\sigma}$. Then, for $\varepsilon>0$ and all $n, m \geq n_{0}$ for some $n_{0} \in \mathbb{N}$,

$$
\left|f_{n}(x)-f_{m}(x)\right|=r\left(\left(f_{n}-f_{m}\right)(x)\right) \leq\left\|f_{n}-f_{m}\right\|_{\sigma} r(x)<\varepsilon r(x) \quad(x \in E) .
$$


This implies that $\left(f_{n}\right)_{n \in \mathbb{N}}$ converges pointwise to a functional $f$ on $E$ and, for all $x$ with $r(x) \leq 1, r\left(\left(f_{n}-f\right)(x)\right) \leq \varepsilon$ for all $n \geq n_{0}$. Hence, $\left\|f_{n}-f\right\|_{\sigma} \leq \varepsilon$, $n \geq n_{0}$, and $\|f\|_{\sigma}=\lim _{n}\left\|f_{n}\right\|_{\sigma}$, which shows that $f \in E^{\sigma}$ and $\left(f_{n}\right)_{n \in \mathbb{N}}$ converges to $f$ in $E^{\sigma}$.

Let $E^{\prime}$ denote the dual space of a normed space $E$. By Proposition 2.9, we have a contractive embedding of $E^{\sigma}$ into $E^{\prime}$, whenever $E$ is spectrally normed. By Example 2.7, this embedding is in general not surjective. However, combining Proposition 2.9 with the above result immediately gives the following special case.

Corollary 3.3. Let $E$ be a spectrally normed space such that $E \subseteq$ $C(X)$. Then $E^{\sigma}$ and $E^{\prime}$ are isometrically isomorphic via the identical mapping.

However, if $E$ is simply a commutative spectrally normed space, the subspace $\operatorname{SB}(E, \mathbb{C})$ of $E^{\prime}$ may be proper and non-closed, as the following example illustrates.

EXAmple 3.4. By the closed graph theorem it is clear that $\operatorname{SB}(E, \mathbb{C})$ is closed in $E^{\prime}$ if and only if $\|\cdot\|$ and $\|\cdot\|_{\sigma}$ are equivalent on $E^{\sigma}$. Therefore our task is to produce an example of a spectrally normed space $E$ and an unbounded sequence $\left(f_{n}\right)_{n \in \mathbb{N}}$ in $E^{\sigma}$ which is bounded in $E^{\prime}$.

Let $c_{0}$ be the non-unital Banach algebra of all complex null sequences. Consider the non-trivial ideal $J$ of $c_{0}$ defined by

$$
J=\left\{\left(x_{k}\right)_{k \in \mathbb{N}} \in c_{0}\left|\sup _{k \in \mathbb{N}}\right| k x_{k} \mid<\infty\right\} .
$$

We define on $J$ a new norm by $\left\|\left(x_{k}\right)_{k \in \mathbb{N}}\right\|^{\prime}=\sup _{k \in \mathbb{N}}\left|k x_{k}\right|$. With this norm, $J$ becomes a commutative Banach algebra under componentwise multiplication. Let $A$ be the unitisation of $J$. It is easy to see that, for each $x=\left(x_{k}\right)_{k \in \mathbb{N}}+x_{0} \in A$, the spectrum is given by $\sigma(x)=\left\{x_{0}, x_{0}+x_{k} \mid k \in \mathbb{N}\right\}$. Consequently, $r(x)=\sup _{k \in \mathbb{N}}\left\{\left|x_{0}\right|,\left|x_{0}+x_{k}\right|\right\}$. In particular, $A$ is semisimple.

Let $T$ be the bounded operator defined by

$$
T: A \rightarrow \ell^{\infty}, \quad\left(x_{k}\right)_{k \in \mathbb{N}}+x_{0} \mapsto\left(\sqrt{k} x_{k}\right)_{k \in \mathbb{N}} .
$$

For every $n \in \mathbb{N}$, let $P_{n}$ be the projection of $\ell^{\infty}$ onto the subspace spanned by the first $n$ canonical unit vectors $e_{1}, \ldots, e_{n}$ and let $T_{n}=P_{n} \circ T$. Then

$r\left(T_{n} x\right)=\sup \left\{\left|\sqrt{k} x_{k}\right| \mid 1 \leq k \leq n\right\} \leq \sqrt{n} \sup \left\{\left|x_{k}\right| \mid 1 \leq k \leq n\right\} \leq 2 \sqrt{n} r(x)$ for all $x \in A$ and $n \in \mathbb{N}$. Therefore, $T_{n} \in \mathrm{SB}\left(A, \ell^{\infty}\right) \subseteq B\left(A, \ell^{\infty}\right)$ for every $n \in \mathbb{N}$.

Clearly, $\left(T_{n}\right)_{n \in \mathbb{N}}$ converges to $T$ in norm. However, $T$ is not spectrally bounded since $T \widetilde{e}_{n}=\sqrt{n} e_{n}$, where $\widetilde{e}_{n}$ denotes the $n$th canonical unit vector in $c_{0}$. 
We have thus shown that $\mathrm{SB}\left(A, \ell^{\infty}\right)$ is not closed in the Banach space $B\left(A, \ell^{\infty}\right)$ and we shall deduce from this that $\operatorname{SB}(A, \mathbb{C})$ is not closed in $A^{\prime}$.

Choose a real number $1<\theta<3 / 2$ and define $g \in\left(\ell^{\infty}\right)^{\prime}$ by

$$
g(x)=\sum_{k=2}^{\infty} k^{-\theta} x_{k-1} \quad\left(x=\left(x_{k}\right)_{k \in \mathbb{N}} \in \ell^{\infty}\right) .
$$

By Corollary 3.3, $g$ is spectrally bounded. Therefore, $f_{n}=T_{n}^{\prime} g \in \operatorname{SB}(A, \mathbb{C})$ for all $n \in \mathbb{N}$. Let $f=T^{\prime} g=\lim _{n} f_{n} \in A^{\prime}$. To see that $f \notin \mathrm{SB}(A, \mathbb{C})$, let $y_{n}=\sum_{k=1}^{n} \widetilde{e}_{n} \in A$. Then $r\left(y_{n}\right)=1$ but

$$
f\left(y_{n}\right)=\sum_{k=2}^{n} k^{-\theta+1 / 2},
$$

which grows arbitrarily large as $n \rightarrow \infty$.

REMARK 3.5. Of course, we have to check that the spectral dual $E^{\sigma}$ is an object in our new category. Since $E^{\sigma}$ is a normed space, it carries the spectral structure inherited from the embedding into $C\left(\left(E^{\sigma}\right)_{1}^{\prime}\right)$; compare Example 2.1.1. Indeed, $\|f\|_{\sigma}=r(f)$ for all $f \in E^{\sigma}$ in this spectral structure. We shall always consider $E^{\sigma}$ as a spectrally normed space endowed with this (canonical) spectral structure.

From Remark 3.5 and Corollary 3.3 we derive the following rigidity property of the spectral dual space construction.

Corollary 3.6. For every spectrally normed space $E$, the dual $\left(E^{\sigma}\right)^{\prime}$ of the spectral dual $E^{\sigma}$ of $E$ is isometrically isomorphic to the spectral bidual $E^{\sigma \sigma}$ of $E$ (via the identical mapping).

COROLLARY 3.7. The adjoint $T^{\sigma}$ of a spectrally bounded operator $T$ is spectrally bounded with $\left\|T^{\sigma}\right\|_{\sigma} \leq\|T\|_{\sigma}$.

Proof. Let $T: E \rightarrow F$ be a spectrally bounded operator between the spectrally normed spaces $E$ and $F$. Denote by $T^{\sigma}: F^{\sigma} \rightarrow E^{\sigma}$ the adjoint mapping $T^{\sigma} g=g \circ T, g \in F^{\sigma}$. By the definition of the dual spectral structure we find

$$
r\left(T^{\sigma} g\right)=\|g \circ T\|_{\sigma} \leq\|g\|_{\sigma}\|T\|_{\sigma}=\|T\|_{\sigma} r(g)
$$

for all $g \in F^{\sigma}$, where we have used Proposition 2.4(iii). As a result, $T^{\sigma} \in$ $\operatorname{SB}\left(F^{\sigma}, E^{\sigma}\right)$ and $\left\|T^{\sigma}\right\|_{\sigma} \leq\|T\|_{\sigma}$.

Before becoming too optimistic about the role of the spectral dual, let us describe it in some non-commutative setting.

ExAmple 3.8. Let $A=M_{n}(\mathbb{C})$ for some $n \in \mathbb{N}$. Take $f \in A^{\sigma}$; then $f$ vanishes on all nilpotent matrices. It is well known that the space $s \ell_{n}(\mathbb{C})$ of all trace zero matrices is linearly spanned by the nilpotent matrices. Denot- 
ing by tr the normalised trace on $A$, we thus find ker $\operatorname{tr} \subseteq \operatorname{ker} f$. It follows that $f=\alpha$ tr for some $\alpha \in \mathbb{C}$. Therefore, $M_{n}(\mathbb{C})^{\sigma}=\mathbb{C}$ independent of $n$.

It even can come worse, as the next example shows.

ExAmple 3.9. Let $A=B(H)$ for some infinite-dimensional Hilbert space $H$. Take $f \in A^{\sigma}$. It is known that every element in $B(H)$ is a sum of nilpotent elements (in fact, elements with square zero); see, e.g., [14, Theorem 2]. As $f$ vanishes on nilpotent elements, $f=0$. Therefore, $B(H)^{\sigma}=\{0\}$.

These examples can be extended as follows. For every properly infinite von Neumann algebra $A, A^{\sigma}=\{0\}$; see [13, Corollary 3.9]. On the other hand, if $A$ is a finite factor with canonical trace $\tau$, then the same arguments as in Example 3.8 apply since the subspace spanned by the nilpotent elements is dense in $\operatorname{ker} \tau$ and every $f \in A^{\sigma}$ is bounded; hence, $f$ is a multiple of $\tau$. It follows that $A^{\sigma}=\mathbb{C}$ in this case as well. However, this does not extend to arbitrary finite von Neumann algebras. If $A=C(X)$, by Corollary $3.3, C(X)^{\sigma}=C(X)^{\prime}$.

The above examples drastically rule out any hope for a Hahn-Banach type theorem for spectrally bounded functionals. Behind this is the fact that, in non-commutative algebras, there may be quasi-nilpotent elements $x$ and $y$ whose sum $z$ is not quasi-nilpotent. If $f$ is a spectrally bounded functional on the one-dimensional subspace spanned by $z$ such that $f(z)=1$, no extension of $f$ to the entire algebra will be spectrally bounded.

Remark 3.10. Let $E \subseteq A$, where the Banach algebra $A$ is commutative modulo the radical. Then the spectral radius is subadditive. Hence, whenever $f \in E^{\sigma}$ we can apply Banach's extension theorem to $f$ with the seminorm $p(x)=\|f\|_{\sigma} r(x), x \in E$. Therefore, an extension $\widetilde{f} \in A^{\sigma}$ of $f$ with the property $\|f\|_{\sigma}=\|\widetilde{f}\|_{\sigma}$ exists. In this situation, all the familiar results in duality theory hold; e.g., $E^{\sigma}$ separates the points in $E$.

Since there is no extension theorem for spectrally bounded functionals in general, criteria which entail spectral boundedness become very important. The following one is a slight modification of an observation made in the commutative case in [2, Lemma 4.1.15]. By co $K$ we denote the convex hull of a subset $K$ of a vector space.

Proposition 3.11. Let $E \subseteq A$ be a spectrally normed space which contains the identity of $A$. Let $f$ be a linear functional on $E$, and let $M>0$. Then $f$ is spectrally bounded with $\|f\|_{\sigma} \leq M=f(1)$ if and only if $f(x) \in$ $M \operatorname{co} \sigma(x)$ for all $x \in E$.

Proof. We first prove the sufficiency of the criterion. If $f(1) \in M \operatorname{co} \sigma(1)$ $=\{M\}$ then $f(1)=M$. From the assumption $f(x) \in M \operatorname{co} \sigma(x)$ it follows directly that $|f(x)| \leq M r(x)$ for each $x \in E$, wherefore $\|f\|_{\sigma} \leq M$. 
To show the necessity, we put $f^{\prime}=f / M$; then $f^{\prime}(1)=1$ and $\left\|f^{\prime}\right\|_{\sigma} \leq 1$. Take $\lambda \notin \operatorname{co} \sigma(x)$. Then

$$
\left|f^{\prime}(x)-\lambda\right|=\left|f^{\prime}(x-\lambda)\right| \leq r(x-\lambda),
$$

which implies that $f^{\prime}(x) \in B(\lambda ; r(x-\lambda))$, the closed disk about $\lambda$ with radius $r(x-\lambda)$. Since $\operatorname{co} \sigma(x)=\bigcap_{|\lambda|>r(x)} B(\lambda ; r(x-\lambda))$, it follows that $f^{\prime}(x) \in \operatorname{co} \sigma(x)$ for all $x \in E$. This yields the assertion.

4. Spectral isometries. Contractions and isometries play a distinguished role in the theory of bounded operators; thus it is not surprising that their spectral relatives deserve special attention as well.

Definition 4.1. Let $E$ and $F$ be spectrally normed spaces. A linear operator $T \in \mathrm{SB}(E, F)$ is said to be a spectral contraction if $\|T\|_{\sigma} \leq 1$, and $T$ is called a spectral isometry if $r(T x)=r(x)$ for all $x \in E$.

Unlike isometries, spectral isometries may only be injective modulo the radical.

Proposition 4.2. Let $T: A \rightarrow B$ be a spectral isometry. Then $\operatorname{ker} T \subseteq$ $\operatorname{rad}(A)$. Thus, $T$ is injective if $A$ is semisimple.

Proof. Suppose $a \in \operatorname{ker} T$. Then, for all $x \in A$, we have

$$
r(x)=r(T x)=r(T x+T a)=r(x+a),
$$

which implies that $a \in \operatorname{rad}(A)$ by [2, Theorem 5.3.1].

Proposition 4.3. Let $T: A \rightarrow B$ be a surjective spectral isometry. Then $T \mathcal{Z}(A) \subseteq \mathcal{Z}(B)$

Proof. The argument relies on the following observation by Pták ([15, Proposition 2.1], see also [2, Theorem 5.2.2]): An element $z$ belongs to the centre modulo the radical if and only if $r(x+z) \leq r(x)+r(z)$ for all $x$.

Take $a \in \mathcal{Z}(A)$, and for $y \in B$ take $x \in A$ such that $T x=y$. Then

$$
r(y+T a)=r(T(x+a))=r(x+a) \leq r(x)+r(a)=r(y)+r(T a) ;
$$

hence $T a \in \mathcal{Z}(B)$.

The operator $T$ is called unital if $T 1=1$. The above results together with a classical theorem enable us to deduce that unital surjective spectral isometries are well behaved on the commutative part of semisimple Banach algebras.

Corollary 4.4. Let $T: A \rightarrow B$ be a unital surjective spectral isometry, and let both $A$ and $B$ be semisimple. Then $T Z(A)=Z(B)$. Furthermore, the restriction $\left.T\right|_{Z(A)}$ is an algebra isomorphism.

Proof. By Proposition 4.3, $T Z(A) \subseteq Z(B)$ and, since $T$ is injective by Proposition 4.2 , we can apply the same argument to the inverse spectral 
isometry $T^{-1}$. Hence, $T Z(A)=Z(B)$. The restriction $\left.T\right|_{Z(A)}: Z(A) \rightarrow$ $Z(B)$ is thus a unital surjective spectral isometry between semisimple commutative Banach algebras $(\operatorname{as} \operatorname{rad}(Z(A))=\operatorname{rad}(A) \cap Z(A))$. By Nagasawa's theorem [2, Theorem 4.1.17], it follows that $\left.T\right|_{Z(A)}$ is an isomorphism.

REMARK 4.5. In some favourable circumstances, the assumption $T 1=1$ in Corollary 4.4 above reduces to a scaling. For instance, if $B=B(E)$ for a Banach space $E$, every spectral isometry $T$ from a semisimple Banach algebra $A$ onto $B$ satisfies $T 1=\lambda 1$ for some unimodular $\lambda \in \mathbb{C}$. The argument used in [10, Theorem 1] for the case $A=B=B(E)$ takes over almost verbatim.

There is some evidence that spectral isometries are well compatible with the algebraic structure, even in a non-commutative environment. It is shown in [10] that every unital surjective spectral isometry on $B(E)$, $E$ a Banach space, is a Jordan isomorphism. This had been obtained for finite-dimensional $E$ earlier by Aupetit [3, Proposition 2]. Sourour proved in [20] that every surjective invertibility-preserving operator $T$ on $B(E)$ satisfies the Jordan property $T\left(x^{2}\right)=(T x)^{2}$ for all $x$. Very recently, Aupetit [4] showed that every surjective spectrum-preserving operator between von Neumann algebras also has this property, thus solving a long-standing problem by Kaplansky in this framework. If $T$ is a spectral isometry on a von Neumann algebra, the results in [13] show that the restriction of $T$ to the properly infinite part (which is a spectrally bounded operator) is a Jordan homomorphism. Taking all these results together, we propose the following conjecture.

CONJeCTURe 4.6. Every unital surjective spectral isometry between unital $C^{*}$-algebras is a Jordan isomorphism.

One of the first cases that has to be studied to make progress on this conjecture is a $\mathrm{II}_{1}$ factor. We also note that dropping "spectral" in Conjecture 4.6 leads to Kadison's celebrated characterisation of Jordan *-isomorphisms.

One of the possible strategies to tackle this problem is to show that spectral isometries preserve large enough parts of the spectrum, in order to apply some of the previous results. We conclude this paper with two rather elementary observations of this type. The first one extends an argument in the proof of [10, Theorem 1] from the case $A=B=B(E)$.

Proposition 4.7. Every bijective unital spectral isometry between two Banach algebras preserves the peripheral spectrum.

Proof. Suppose that $T: A \rightarrow B$ is a bijective unital spectral isometry between the Banach algebras $A$ and $B$. Let $a \in A$ and take $\lambda \in \sigma(a)$ with 
$|\lambda|=r(a)$. Then

$$
r(T a+\lambda)=r(T(a+\lambda))=r(a+\lambda)=2|\lambda|=2 r(a) .
$$

Hence, there is $\alpha \in \sigma(T a)$ such that $|\alpha+\lambda|=2|\lambda|$, wherefore $|\alpha| \geq|\lambda|$. On the other hand, $|\alpha| \leq r(T a)=r(a)=|\lambda|$ so that $|\alpha|=|\lambda|$. From

$$
|\alpha-\lambda|^{2}=2|\alpha|^{2}+2|\lambda|^{2}-|\alpha+\lambda|^{2}=4|\lambda|^{2}-(2|\lambda|)^{2}=0
$$

we thus deduce that $\alpha=\lambda$. Therefore, $\lambda$ belongs to the peripheral spectrum of $T a$. The same argument applied to $T^{-1}$ yields the reverse inclusion.

Proposition 4.8. Every unital spectral isometry between two Banach algebras preserves the convex hull of the spectrum.

Proof. Suppose that $T: A \rightarrow B$ is a unital spectral isometry between the Banach algebras $A$ and $B$. The convex hull $\operatorname{co} \sigma(a)$ of the spectrum of an element $a$ is the intersection of all disks $B(\lambda ; r(a-\lambda)),|\lambda|>r(a)$. Therefore,

$$
\operatorname{co} \sigma(T a)=\bigcap_{|\lambda|>r(T a)} B(\lambda ; r(T a-\lambda))=\bigcap_{|\lambda|>r(a)} B(\lambda ; r(a-\lambda))=\operatorname{co} \sigma(a) .
$$

In particular, every unital spectral isometry preserves singleton spectra.

\section{References}

[1] B. Aupetit, The uniqueness of the complete norm topology in Banach algebras and Banach Jordan algebras, J. Funct. Anal. 47 (1982), 1-6.

[2] - A Primer on Spectral Theory, Springer, New York, 1991.

[3] - Spectral characterization of the radical in Banach and Jordan-Banach algebras, Math. Proc. Cambridge Philos. Soc. 114 (1993), 31-35.

[4] - Spectrum-preserving linear mappings between Banach algebras or Jordan-Banach algebras, J. London Math. Soc. 62 (2000), 917-924.

[5] B. Aupetit and M. Mathieu, The continuity of Lie homomorphisms, Studia Math. 138 (2000), 193-199.

[6] M. I. Berenguer and A. R. Villena, Continuity of Lie isomorphisms of Banach algebras, Bull. London Math. Soc. 31 (1999), 6-10.

[7] R. Bhatia, P. Šemrl and A. R. Sourour, Maps on matrices that preserve the spectral radius distance, Studia Math. 134 (1999), 99-110.

[8] M. Brešar, Derivations decreasing the spectral radius, Arch. Math. (Basel) 61 (1993), 160-162.

[9] M. Brešar and M. Mathieu, Derivations mapping into the radical, III, J. Funct. Anal. 133 (1995), 21-29.

[10] M. Brešar and P. Šemrl, Linear maps preserving the spectral radius, ibid. 142 (1996), 360-368.

[11] R. E. Curto and M. Mathieu, Spectrally bounded generalized inner derivations, Proc. Amer. Math. Soc. 123 (1995), 2431-2434.

[12] M. Mathieu, Where to find the image of a derivation, in: Banach Center Publ. 30, Inst. Math., Polish Acad. Sci., Warszawa, 1994, 237-249. 
[13] M. Mathieu and G. J. Schick, Spectrally bounded operators from von Neumann algebras, J. Operator Theory, in press.

[14] C. Pearcy and D. Topping, Sums of small numbers of idempotents, Michigan Math. J. 14 (1967), 453-465.

[15] V. Pták, Derivations, commutators and the radical, Manuscripta Math. 23 (1978), $355-362$.

[16] - Commutators in Banach algebras, Proc. Edinburgh Math. Soc. 22 (1979), 207211.

[17] A. Rodríguez Palacios, The uniqueness of the complete norm topology in complete normed nonassociative algebras, J. Funct. Anal. 60 (1985), 1-15.

[18] G. J. Schick, Spectrally bounded operators on Banach algebras, PhD thesis, Queen's Univ. Belfast, Belfast, 2001.

[19] P. Šemrl, Spectrally bounded linear maps on B(H), Quart. J. Math. Oxford (2) 49 (1998), 87-92.

[20] A. R. Sourour, Invertibility preserving linear maps on $L(X)$, Trans. Amer. Math. Soc. 348 (1996), 13-30.

[21] M. P. Thomas, The image of a derivation is contained in the radical, Ann. of Math. (2) 128 (1988), 435-460.

[22] J. Zemánek, Properties of the spectral radius in Banach algebras, in: Banach Center Publ. 8, PWN, Warszawa, 1982, 579-595.

Department of Pure Mathematics

Queen's University Belfast

Belfast BT7 1NN

Northern Ireland

E-mail: m.m@qub.ac.uk

g.schick@qub.ac.uk

Received January 24, 2002

Revised version March 11, 2002 